\title{
Prevalência de Cândida na Flora Vaginal de Mulheres Atendidas num Serviço de Planejamento Familiar
}

Prevalence of Candida in the Vagina of Women Attended at a Family Planning Service

Antônio Aleixo Neto, Júnia Soares Hamdan, Ressalla Castro Souza

\section{RESUMO}

Objetivo: avaliar a prevalência de Candida sp. e a distribuição de suas espécies na flora vaginal de mulheres numa clinica de planejamento familiar.

Método: estudo transversal no qual se avaliaram prospectivamente 72 mulheres não-grávidas, com ou sem queixas específicas, sendo coletadas amostras de secreção vaginal para cultura de leveduras, efetuada a medição do $\mathrm{pH}$ vaginal e anotados dados de achados do exame ginecológico.

Resultados: leveduras pertencentes ao gênero Candida foram encontradas em 18 casos (25\%). A C. albicans foi a espécie mais prevalente $(77,8 \%)$ e conseqüentemente $22,2 \%$ foram não-albicans. Entre as não-albicans a espécie mais prevalente foi a C. glabrata (16,7\%) seguida pela $C$. parapsilosis (5,6\%). Prurido e ardor foram os únicos sintomas relacionados significativamente com a presença de cândida. Foi observado que a C. glabrata, ao contrário da C. albicans, não causa corrimento clinicamente verificável ao exame ginecológico. Não foram verificadas associações de alguns fatores predisponentes (idade, escolaridade e uso de contraceptivos) com a presença ou não de cândida.

Conclusões: nossos resultados sugerem: a) uma alta prevalência de Candida sp. entre as mulheres (25\%); b) que as espécies não-albicans desempenham um papel importante no meio vaginal; c) que prurido e ardor são os sintomas mais comuns na presença de cândida $e$ d) que a C. glabrata não costuma causar corrimento vaginal ao exame ginecológico. Finalmente, é importante observar que nossos resultados são consistentes com o que a literatura internacional tem mostrado nos últimos anos.

PALAVRAS-CHAVE: Vulvovaginite. Candidiase vaginal. Contracepção oral.

\section{Introdução}

A candidíase vulvovaginal é uma das infecções mais comuns na prática clínica de um ginecologista. Na Inglaterra a incidência varia entre 28 e $37 \%$ das mulheres. Nos Estados Unidos, a incidência desta infecção micótica tem também crescido acentuadamente, sendo a segunda causa de vaginite logo após a vaginose bacteriana ${ }^{1}$. Estima-se que naquele país o número de mulheres

Serviço de Ginecologia e Obstetrícia

Hospital das Clínicas da UFMG

Correspondência:

Antônio Aleixo Neto

R. Nicarágua, 108/102-A

30320-050 - Belo Horizonte - MG

e-mail: aleixo@medicina.ufmg.br acometidas com candidiase vulvovaginal atinja mais de 13 milhões por ano ${ }^{2}$. No Brasil não existem dados epidemiológicos sobre a candidiase.

Já foram identificadas mais de 400 cepas de cândida. Embora a espécie Candida albicans seja a mais importante causadora de candidiase, a incidência de não-albicans tem crescido de maneira importante nos últimos anos. Na década de 70 as espécies não-albicans não eram encontradas em mais do que $5-10 \%$ dos casos de infecção genital. Já na década de 80 a maioria dos trabalhos mostravam um crescimento desta taxa para 15 a $25 \%{ }^{4}$. O aumento da incidência dessas espécies tem importância, na medida em que apresentam sintomatologia mais branda do que a C. albicans e um espectro diferente de resistência aos tratamentos antifúngicos ${ }^{3}$. 
O gênero Candida pode ser isolado no trato genital entre 10 e $55 \%$ das mulheres assintomáticas na idade fértil ${ }^{4}$. Assim, a sua presença em tão larga escala na microbiota vaginal nos permite classificá-la como saprófita, ou seja, a presença desse microorganismo não significa, necessariamente, a presença da doença. Embora seja significativa a prevalência da cândida como constituinte da flora vaginal, é mais provável que o principal reservatório seja o trato gastrintestinal, visto que pode ser encontrada em $56 \%$ das amostras de fezes obtidas ao acaso, em uma população $0^{4}$. A vulvovaginite por cândida raramente é uma doença transmitida sexualmente. Basicamente, o surto aconteceria por um mecanismo endógeno e oportunista ${ }^{4}$.

O objetivo deste estudo foi obter dados de prevalência da Candida sp. e a distribuição de suas espécies na flora vaginal de mulheres numa clínica de planejamento familiar.

\section{Pacientes e Métodos}

Foi realizado um estudo prospectivo, transversal, em que foram incluídas setenta e duas mulheres com idade entre 15 e 54 anos (média: 29,2; DP: 8,2) que consultaram o Serviço de Planejamento Familiar do Hospital das Clínicas da Universidade Federal de Minas Gerais no ano de 1996. A população atendida por este Serviço é constituída de mulheres em idade fértil, nãográvidas, em uso ou desejando usar algum método contraceptivo. Entre as pacientes que já estavam em uso de métodos contraceptivos, a grande maioria era constituída de mulheres que iam fazer as revisões agendadas e uma minoria de mulheres não-agendadas, com alguma queixa ginecológica.

Foi realizada coleta sistemática da secreção vaginal em todas as pacientes que preenchiam os seguintes critérios: não-grávidas, não-menstruadas e que não tivessem utilizado antibióticos orais, fungicidas e cremes vaginais nos últimos trinta dias. Todos os exames ginecológicos e a coleta de dados e do material foram realizados pelo mesmo profissional. A coleta do material foi realizada com "swab" esterilizado, inoculado em ágar Sabouraud e a incubação realizada a $28^{\circ} \mathrm{C}$ por 72 horas. As leveduras isoladas foram identificadas de acordo com sua micromorfologia, provas bioquímicas e teste do tubo germinativo, no Laboratório de Microbiologia do Instituto de Ciências Biológicas da UFMG. A medição de $\mathrm{pH}$ foi efetuada por meio de fitas Universalindikator Merck, com escala unitária de 0-14.

Foram anotados dados sociodemográficos de todas as pacientes selecionadas, além do uso atual de métodos contraceptivos, número de parceiros sexuais, queixas atuais relacionadas com possiveis vaginites, a impressão clínica do médico quanto à secreção vaginal (fisiológica ou não) e a determinação do $\mathrm{pH}$ vaginal.

Para avaliar a prevalência das espécies de Candida e as demais comparações de proporção, foram utilizados o teste estatístico $\chi^{2}$ e o teste de Fisher, quando aplicável. Foi utilizado o programa Epi-Info v6.

\section{Resultados}

Entre as mulheres participantes do estudo, a média de idade foi de 29,2 anos (DP 8,2) e a paridade média de 1,8 filhos (DP 1,1). Oitenta e sete por cento das mulheres eram casadas ou amasiadas e a escolaridade da grande maioria $(86,1 \%)$ era de primário completo ou incompleto (Tabela 1). A maioria das mulheres utilizava algum método contraceptivo $(86,1 \%)$, sendo o dispositivo intra-uterino o método mais prevalente $(65,3 \%)$, seguido da pílula (13,9\%) (Figura 1).

Tabela 1 - Características sociodemográficas das pacientes submetidas à investigação para detecção de Candida sp.

\begin{tabular}{|c|c|c|}
\hline & $\mathrm{N}$ & $\%$ \\
\hline \multicolumn{3}{|l|}{ Grupo etário } \\
\hline $15-19$ & 3 & 4,2 \\
\hline $20-24$ & 12 & 16,7 \\
\hline $25-29$ & 20 & 27,8 \\
\hline $30-34$ & 14 & 19,4 \\
\hline $35-39$ & 11 & 15,3 \\
\hline $40+$ & 12 & 16,7 \\
\hline \multicolumn{3}{|l|}{ Paridade } \\
\hline 0 & 4 & 5,6 \\
\hline 1 & 27 & 37,5 \\
\hline 2 & 25 & 34,7 \\
\hline 3 & 12 & 16,7 \\
\hline $4+$ & 4 & 5,6 \\
\hline \multicolumn{3}{|l|}{ Estado civil } \\
\hline Casada & 48 & 66,7 \\
\hline Solteira & 7 & 9,7 \\
\hline Amasiada & 15 & 20,8 \\
\hline Separada & 2 & 2,8 \\
\hline \multicolumn{3}{|l|}{ Escolaridade } \\
\hline Nenhuma & 1 & 1,4 \\
\hline $1^{\circ} \mathrm{grau}$ incompleto & 45 & 62,5 \\
\hline $1^{\circ}$ grau completo & 17 & 23,6 \\
\hline $2^{\circ}$ grau & 7 & 9,7 \\
\hline Superior & 2 & 2,8 \\
\hline
\end{tabular}




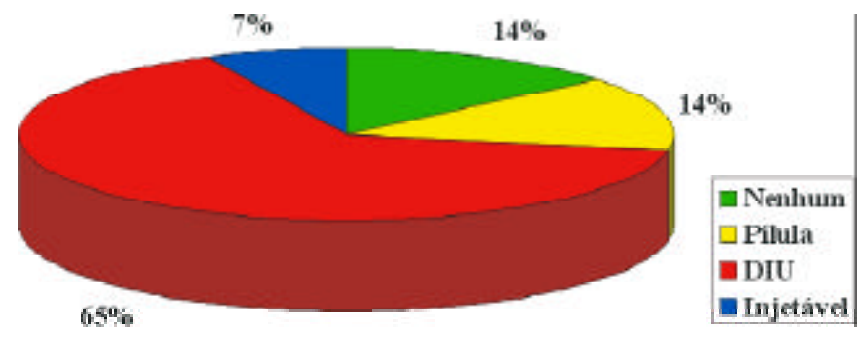

Figura 1 - Distribuição de métodos contraceptivos utilizados.

A grande maioria das mulheres relatou ter apenas um parceiro sexual $(95,8 \%)$, sendo que apenas uma relatou ter dois parceiros.

Foram encontrados 18 casos positivos para cândida (25\%), sendo a C. albicans a espécie mais prevalente $(77,8 \%)$ e $22,2 \%$ de não-albicans. Entre as não-albicans a espécie mais prevalente foi a $C$. glabrata $(16,7 \%)$ seguida pela $C$. parapsilosis $(5,6 \%)$ (Tabela 2).

Tabela 2 - Distribuição das espécies de Candida em pacientes submetidas à investigação para detecção de Candida sp.

\begin{tabular}{lcc}
\hline Espécie & $\mathbf{n}$ & $\%$ \\
\hline C. albicans & 14 & 77,8 \\
C. glabrata & 3 & 16,7 \\
C. parapsilosis & 1 & 5,6 \\
\hline
\end{tabular}

As mulheres com a presença de cândida apresentavam prurido e ardor do com mais freqüência do que as não-portadoras, com razão de risco de 4,1 (IC 95\% 2,02 - 8,48) e 3,1 (IC 95\% 1,51-6,54), respectivamente. Já a queixa de corrimento e dispareunia não estiveram relacionadas com a presença de cândida (Tabela 3).

Tabela 3 - Associação de sinais e sintomas com a presença de cândida

\begin{tabular}{llrl}
\hline Sinais $/$ & Candida & \multicolumn{1}{l}{ Candida ausente } & \\
\hline sintomas & presente & & \\
Prurido & $9 / 18(50,0 \%)$ & $9 / 54(16,7 \%)$ & $p=0,0005^{*}$ \\
Corrimento & $8 / 18(44,4 \%)$ & $17 / 54(31,5 \%)$ & $p=0,4$ \\
Ardor & $4 / 18(22,2 \%)$ & $2 / 54(3,7 \%)$ & $p=0,03$ * \\
Dispareunia & $3 / 18(16,7 \%)$ & $4 / 54(7,4 \%)$ & $p=0,3$ \\
\hline
\end{tabular}

* Significativo

Por outro lado, quando se compara a presença de prurido entre as portadoras de C. albicans ou da C. glabrata verifica-se uma maior incidência deste sintoma entre as primeiras $(57,1 \%$ e $33,3 \%$, respectivamente), no entanto, sem significância estatística $(p=0,58)$. A $C$. parapsilosis não foi analisada, em razão do pequeno número (apenas um caso).

Ao exame ginecológico observou-se que a maioria das pacientes com cândida $(77,8 \%)$ apresentava ao exame ginecológico uma secreção vaginal anormal, geralmente de cor branca. Quando se analisou por espécie, verificou-se que nenhum dos casos de C. glabrata apresentou ao exame sinais de secreção vaginal anômala. Ao contrário, a grande maioria das portadoras de C. albicans $(92,8 \%)$ apresentava corrimento suspeito.

Em 71 mulheres foi medido o $\mathrm{pH}$ vaginal e a análise para tendência linear não mostrou associação significativa entre os valores de $\mathrm{pH}$ vaginal e a presença de cândida (Tabela 4).

Tabela 4 - Associação do pH vaginal com a presença de cândida.

\begin{tabular}{cccc}
\hline $\mathbf{p H}$ & Candida + & Candida - & Total \\
\hline 4 & 6 & 21 & 27 \\
5 & 8 & 13 & 21 \\
6 & 1 & 6 & 7 \\
7 & 2 & 9 & 11 \\
8 & 1 & 3 & 4 \\
9 & 0 & 1 & 1 \\
Total & 18 & 53 & 71 \\
\hline Análise para tendência linear & & \\
$p=0,6$ & &
\end{tabular}

Foram analisados alguns fatores de risco potenciais para a presença de cândida (Tabela 5). As mulheres foram categorizadas em grupos de cinco anos. Embora tenha sido encontrada uma tendência para positividade da cultura para cândida com o acréscimo da idade, este achado não foi significativo. Também a escolaridade e o uso de diversos métodos contraceptivos não afetaram a prevalência de cândida.

\section{Discussão}

O delineamento desse estudo objetivou avaliar a prevalência de cândida na microbiota vaginal de mulheres supostamente hígidas ou com queixas e também mostrar a importância das espécies não-albicans na atualidade. Este interesse se explica pela carência de dados epidemiológicos sobre esse assunto em nosso meio. Por isso, o diagnóstico e a conduta terapêutica dos médicos nos casos de candidiase vulvovaginal têm ficado muitas vezes na dependência de estimativas individuais e da experiência pessoal. Embora o estudo tenha incluido portadoras de cândida com 
ou sem sintomas, a tendência mais moderna tem sido considerar ambas como portadoras de candidiase vulvovaginal. Está inerente nesta definição que o aparecimento dos sintomas é uma questão de tempo e é função da resposta do hospedeiro à ação do microorganismo ${ }^{5}$. O fato de o estudo ter sido realizado num ambulatório de planejamento familiar e não em uma clínica de DST ou de referência minimiza a possibilidade de ter ocorrido um viés de seleção.

Tabela 5 - Avaliação de alguns fatores de risco para isolamento de Candida sp.

\begin{tabular}{|c|c|c|c|}
\hline & Candida + & Candida - & Total \\
\hline \multicolumn{4}{|l|}{ Grupo etário } \\
\hline $15-19$ & 1 & 5 & 6 \\
\hline $20-24$ & 4 & 15 & 19 \\
\hline $25-29$ & 1 & 14 & 15 \\
\hline $30-34$ & 7 & 9 & 16 \\
\hline \multirow[t]{2}{*}{$35+$} & 5 & 11 & 16 \\
\hline & & Análise para tendência linear & $p=0,15$ \\
\hline \multicolumn{4}{|l|}{ Escolaridade } \\
\hline Nenhuma & 1 & 0 & 1 \\
\hline $1^{\circ}$ grau incompleto & 9 & 36 & 45 \\
\hline $1^{\circ}$ grau completo & 4 & 13 & 17 \\
\hline $2^{\circ}$ grau & 4 & 3 & 7 \\
\hline \multirow[t]{2}{*}{ Superior } & 0 & 2 & 2 \\
\hline & & Análise para tendência linear & $p=0,49$ \\
\hline \multicolumn{4}{|l|}{ Contraceptivo } \\
\hline Nenhum & 0 & 10 & 10 \\
\hline Anticoncepcional oral & 4 & 6 & 10 \\
\hline DIU & 12 & 35 & 47 \\
\hline \multirow[t]{2}{*}{ Injetáveis } & 2 & 3 & 5 \\
\hline & & Análise para tendência linear & $p=0,16$ \\
\hline
\end{tabular}

Os resultados desse estudo mostraram que entre mulheres atendidas numa clínica de planejamento familiar a prevalência de cândida na flora vaginal chega a $25 \%$ e que as espécies nãoalbicans atingem a 22,2\% desses casos. Existem diversos trabalhos mostrando uma similaridade com nossos achados. Sobel ${ }^{2}$ relata que a cândida pode ser isolada em $20 \%$ das mulheres assintomáticas em idade reprodutiva. Eckert et al. ${ }^{6}$ encontraram uma prevalência de $24 \%$ entre 774 mulheres com queixas especificas numa clínica de DST nos EUA. Spinillo et $\mathrm{al}^{7}$ isolaram espécies de Candida em $34,5 \%$ das pacientes sintomáticas na Universidade de Pavia, na Itália. Os mesmos autores observaram uma prevalência de 17,2\% de vaginite fúngica causada pelas espécies nãoalbicans em 1995 contra apenas 9,9\% em 1988, denotando uma aumento significativo. Horowitz et al. ${ }^{5}$, numa revisão de sete estudos na década de 80 , mostraram uma prevalência de $21,3 \%$ de espécies não-albicans. Assim como relatam Sobel et al. ${ }^{8}$, encontramos a C. glabrata como a espécie não-albicans mais prevalente $(16,7 \%)$. A outra espécie encontrada, a $C$. parapsilosis, não é tão relatada como das mais prevalentes.

Diversas opiniões têm sido emitidas em relação à prevalência relativamente alta das nãoalbicans. Spinillo et al. ${ }^{7}$ aventam a possibilidade do uso errôneo, inadequado ou incompleto dos antifúngicos, o que possibilitaria a eliminação das C. albicans mais sensiveis, selecionando as nãoalbicans mais resistentes. Horowitz et al. ${ }^{5}$ relatam que diversos agentes antifúngicos, tais como clotrimazol, cetoconazol, miconazol, nistatina e butoconazol, não são ativos in vitro ou in vivo contra as espécies não-albicans.

As queixas de prurido e ardor foram significativamente relacionadas com a colonização por cândida, ao contrário do corrimento e da dispareunia. Eckert et al. ${ }^{6}$ também mostraram que a queixa de corrimento não foi específica para cândida (somente $57 \%$ de especificidade, com um valor preditivo positivo de $26 \%$ ). Sobel et al. ${ }^{8}$ advertem que nenhum dos sintomas de candidiase 
vulvovaginal pode ser considerado patognomônico. Assim como Geiger et al. ${ }^{9}$, não encontramos diferença significativa entre a $C$. albicans e a $C$. glabrata, quanto à queixa de prurido.

$\mathrm{O}$ achado de corrimento vaginal ao exame ginecológico mostrou-se um dado útil para o diagnóstico diferencial entre as espécies de Candida, uma vez que $92,8 \%$ das mulheres em que foi visibilizado um corrimento anormal eram portadoras de C. albicans. Em nenhuma das mulheres portadoras de C. glabrata foi encontrada suspeita de corrimento patológico ao exame. Isto pode significar que as não-albicans podem passar despercebidas ao exame, apesar das queixas. Considerando-se que o exame a fresco também não é adequado para identificar outras espécies de Candida além da albicans, pelo fato de estas não produzirem hifas ${ }^{5,6,8}$, podemos chegar à conclusão de que o exame de cultura para fungos deveria desempenhar, hoje, um papel mais importante no diagnóstico e tratamento da candidíase vulvovaginal.

$\mathrm{O} \mathrm{pH}$ vaginal deve permanecer normal na presença de cândida ${ }^{8}$. No nosso estudo utilizamos fitas de medição com escala unitária, ou seja, não tinham sensibilidade para captar variações menores que 1 no $\mathrm{pH}$. Talvez em virtude disto, a análise de tendência linear não mostrou associação entre os valores de $\mathrm{pH}$ e a presença ou não de cândida.

Dos possiveis fatores de risco analisados (idade, escolaridade e uso de contraceptivos), não encontramos nenhuma associação significativa com a presença de cândida na cavidade vaginal. Eckert et al. ${ }^{6}$ encontraram uma diminuição da positividade da cultura para cândida com o aumento da idade. Os mesmos autores, como nós, não encontraram associação entre o uso de anticoncepcionais orais e a presença de cândida. $\mathrm{Na}$ realidade, esta associação apresenta relatos inconsistentes na literatura. Acreditamos, como Horowitz et al. ${ }^{5}$, ser mais provável que os anticoncepcionais orais com dose mais baixa de estrógenos, os mais utilizados na atualidade, tenham pouca influência como fator predisponente.

Acreditamos que os dados aqui mostrados possam ajudar na avaliação diagnóstica e terapêutica dessa patologia tão prevalente que é a candidiase vulvovaginal.

\section{SUMMARY}

Purpose: to estimate the prevalence of Candida sp. and the distribution of its species in the vagina of women attended at a family planning Service.

Methods: a cross-sectional study evaluating prospectively 72 nonpregnant women, with or without specific complaints.
Samples were checked for the presence of yeast and vaginal $p H$. Data obtained by gynecologic examination were also recorded.

Results: yeast belonging to the genus Candida was isolated from $18(25 \%)$ patients. The most prevalent species was $C$. albicans $(77,8 \%)$ and therefore, $22.2 \%$ were non-albicans species. The most prevalent among the non-albicans species was C. glabrata (16.7\%) followed by C. parapsilosis (5.6\%). The symptoms most directly associated with Candida were itching and burning. Different from the symptoms directly associated with $C$. albicans infection, we observed that $C$. glabrata does not cause vaginal discharge. There was no positive association between predisponent factors such as age, education and contraceptive method used and the presence or absence of Candida.

Conclusions: our results suggest: (i) that there is a high prevalence of Candida species among women; (ii) that nonalbicans species play an important role in the vaginal medium; (iii) that itching and burning are the most common symptoms in women with Candida, and (iv) C. glabrata usually does not cause vaginal discharge. Finally, it is important to emphasize that our results are consistent with the present worldwide findings.

KEY WORDS: Vulvovaginitis. Candida albicans. Contraceptives, oral.

\section{Referências}

1. Kent HL. Epidemiology of vaginitis. Am J Obstet Gynecol $1991 ; 165: 1168-76$.

2. Sobel JD. Candidal Vulvovaginitis. Clin Obstet Gynecol 1993; 36:153-65.

3. Working Group of the British Society for Medical Mycology. Management of genital candidiasis. Br Med J 1995, 310:1241-4.

4. Monif G. Manual de doenças infecciosas em Ginecologia e Obstetrícia. $4^{a}$ ed. Porto Alegre: Artes Médicas, p.114-32.

5. Horowitz BJ, Giaquinta D, Ito S. Evolving pathogens in vulvovaginal candidiasis: implications for patient care. J Clin Pharmacol 1992; 32:248-55.

6. Eckert LO, Hawes SE, Stevens CE, Koutsky LA, Eschenbach DA, Holmes KK. Vulvovaginal candidiasis: clinical manifestations, risk factors, management algorithm. Obstet Gynecol 1998; 92:757-65.

7. Spinillo A, Capuzzo E, Gulminetti R, Marone P, Colonna L, Piazzi G. Prevalence of and risk factors for fungal vaginitis caused by non-albicans species. Am J Obstet Gynecol 1997; 176:138-41.

8. Sobel JD, Faro S, Force RW, Foxman B, Ledger WJ, Nyirjesy $\mathrm{PR}$, et al. Vulvovaginal candidiasis: epidemiologic, diagnostic, and therapeutic considerations. Am J Obstet Gynecol 1998; 178:203-11.

9. Geiger AM, Foxman B, Sobel JD. Chronic vulvovaginal candidiasis: characteristics of women with Candida albicans, C glabrata and no candida. Genitourin Med 1995; 71:304-7. 